\title{
Barriers and Facilitators Along the HIV Care Cascade Among Hospitalized Adolescents in Western Kenya
}

\author{
Marielle Cabe ${ }^{1}$, Judith Toromo ${ }^{2,3}$, Leslie A. Enane ${ }^{2,3}$ \\ ${ }^{1}$ Indiana University School of Medicine, ${ }^{2}$ Academic Model Providing Access To Healthcare \\ (AMPATH), Eldoret, Kenya, ${ }^{3}$ The Ryan White Center for Pediatric Infectious Disease and Global \\ Health, Department of Pediatrics, Indiana University School of Medicine
}

Background/Objective: There are 1.7 million adolescents living with HIV (ALHIV) globally, the majority of whom live in Eastern and Southern Africa. Despite increasing access to antiretroviral therapy (ART), ALHIV experience poorer HIV-related outcomes at each stage of the care cascade relative to other age groups. We sought to investigate barriers and facilitators experienced at each stage of the HIV "care cascade" - testing, linkage to care, retention in care, and adherence to and viral suppression on ART - among a cohort of hospitalized adolescents.

Methods: This qualitative study used semi-structured interviews of hospitalized ALHIV and their caregivers to investigate if crucial delays occurred at each stage of the HIV care cascade. Interviews explored barriers and facilitators at each stage, and were guided by a framework that considered adolescent- or family-level factors, developmental and mental health needs, societylevel, programmatic, and health system factors. Interviews were audio-recorded, transcribed and translated. Members of the study team independently coded each transcript after intercoder reliability was established.

Results: Hospitalized ALHIV experienced critical delays in care, most frequently at the stages of HIV diagnosis and ART adherence. Common barriers to testing related to lack of awareness around perinatal HIV and unknown maternal HIV status. Barriers to adherence were complex and heterogeneous. Common barriers included lack of HIV disclosure or problematic disclosure to the adolescent; intense stigma and challenges coming to terms with one's HIV status; and issues related to ART side effects and tolerance. Facilitators to adherence included family supervision and support in ART taking, and HIV education and adherence counseling provided at the clinic.

Conclusion and Policy Impact: Hospitalized adolescents experienced significant delays in care, most frequently at the stages of pediatric HIV diagnosis and ART adherence. Barriers to adherence are particularly complex. Interventions to address adolescent ART adherence are urgently needed. 